\title{
Educación virtual multimedia en el contexto de la pandemia del COVID -19: Aspectos positivos y negativos
}

Virtual education in the context of the COVID-19 pandemic: Positives and negatives

1 Luis Miguel Viñan Carrasco

https://orcid.org/0000-0003-2127-4038 Universidad Nacional de Chimborazo, Facultad de Ciencias Políticas y Administrativas, Carrera de Comunicación. Riobamba, Ecuador

luis.vinan@unach.edu.ec

2 Miriam Elizabeth Erazo Rodríguez

iD https://orcid.org/0000-0003-1569-7245 Universidad Nacional de Chimborazo, Facultad de Ciencias Políticas y Administrativas, Carrera de Comunicación. Riobamba, Ecuador miriamerazo@unach.edu.ec

3 Myriam Elizabeth Murillo Naranjo iD https://orcid.org/0000-0002-5141-353X Universidad Nacional de Chimborazo, Facultad de Ciencias Políticas y Administrativas, Carrera de Comunicación. Riobamba, Ecuador myriammurillo@unach.edu.ec

4 Fabián Alfonso Calderón Cruz https://orcid.org/0000-0001-5472-3405 Escuela Superior Politécnica de Chimborazo, Facultad de Informática y Electrónica, Carrera de Diseño Gráfico, Riobamba, Ecuador f_calderon@espoch.edu.ec

Artículo de Investigación Científica y Tecnológica Enviado: 03/11/2021

Revisado: $15 / 11 / 2021$

Aceptado: 22/12/2021

Publicado:05/01/2022

DOI: https://doi.org/10.33262/exploradordigital.v6i1.1962

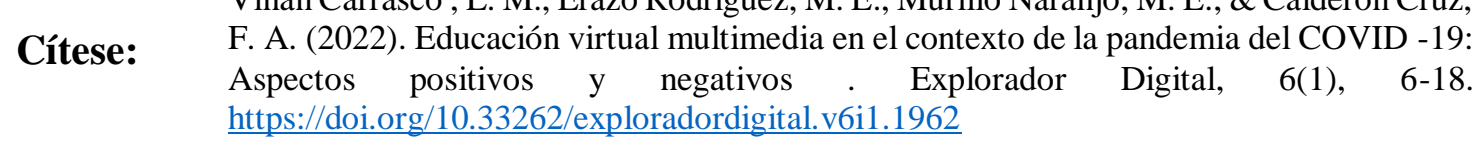

EXPLORADOR DIGITAL, es una Revista electrónica, Trimestral, que se publicará en soporte electrónico tiene como misión contribuir a la formación de profesionales competentes con visión humanística y crítica que sean capaces de exponer sus resultados investigativos y científicos en la misma medida que se promueva mediante su intervención cambios positivos en la sociedad. https://exploradordigital.org

La revista es editada por la Editorial Ciencia Digital (Editorial de prestigio registrada en la Cámara Ecuatoriana de Libro con No de Afiliación 663) www.celibro.org.ec 


Palabras
claves:
educación
virtual; covid-
19; aprendizaje
activo; calidad;
motivación.

\section{Resumen}

Introducción: La pandemia del COVID-19 ha transmutado la vida de las personas a nivel mundial, los cambios son evidentes en todos los ámbitos, y esta nueva normalidad de la que somos parte irrumpe en nuestras actividades cotidianas. La educación es sin duda uno de los campos que ha tenido que realizar modificaciones abruptas en los procesos educativos, las clases presenciales en todos los niveles y ofertas educativas migraron a la modalidad virtual en casi todos los países del mundo. La educación virtual como solución a la emergencia sanitaria por el confinamiento en el que nos vimos inmersos, inclinó su metodología al uso de herramientas digitales en todos los contextos educativos; lo que ha originado investigaciones a nivel internacional, respecto a las percepciones y reflexiones en cuanto a los aspectos negativos y positivos que trae consigo la educación virtual en el contexto del COVID-19, se desconoce qué factores son los más relevantes en la actualidad para los estudiantes y que permitan mejorar la educación virtual en el contexto de la pandemia. Por los motivos señalados la presente investigación presenta resultados de los factores positivos y negativos de la educación virtual en el contexto del covid-19. Objetivo: Exponer resultados de los factores positivos y negativos de la educación virtual en el contexto del COVID -19. Metodología: El estudio tiene un enfoque cuantitativo, descriptivo, diseño no experimental, transaccional, la muestra es de 380 estudiantes de educación superior de Riobamba (nivel de confianza del $95 \%$, error muestral del 5\%). Recogida de datos mediante encuesta online del 28 de junio al 2 de agosto de 2021, instrumento adaptado a la educación virtual, análisis de datos SPSS-25. Las fases de la investigación: solicitud de requerimientos del público objetivo, revisión de la literatura, rediseño del cuestionario (panel de expertos), encuesta online (aplicación), análisis de datos. Resultados: Se observa un acuerdo con la educación virtual, respecto a la atención (1. Interesantes y las actividades virtuales ayudan a mantener la atención), confianza (2. Seguridad para aprender y superar las evaluaciones), satisfacción (3. Disfruto y es un placer aprender en la modalidad virtual), relevancia (4. Los videos didácticos son importantes, las actividades virtuales son importantes para desarrollar mis habilidades y puedo relacionar el contenido con experiencias reales). No se observa indiferencia en los resultados. En desacuerdo con el material didáctico en textos es 


Keywords:
virtual
education,
covid-19, active
learning,
quality,
motivation.

Keywords:

virtual

education, covid-19, active

learning, motivation aburrido, es difícil entender algunas guías instruccionales, la interacción y algunos contenidos no fueron relevantes para mis necesidades. Conclusión: El aprendizaje activo favorece la evaluación positiva de la educación virtual, por otro lado, se deben tomar en cuenta las deficiencias relacionadas con el material didáctico e interacciones interpersonales para mejorarlo, el diseño de actividades de aprendizaje motivadoras para incrementar los niveles de aprendizaje.

\section{Abstract}

Introduction. Speaking is considered one of the most difficult skills to be developed in class when teaching a foreign language. Therefore, teachers need to look for alternatives to enhance learners' oral production. In this regard, Communicative activities (CA) play a fundamental role in language teaching due to the fact they provide them with opportunities to use language as in real situations. Objective. The main goal of this research is to provide a general overview of Communicative activities to enhance oral production in the EFL classroom. Methodology. This article was based on the qualitative approach. A descriptive - documentary research was carried out through an extensive bibliographic literature review about the main principles of the Communicative Approach, communicative activities, types of CA, and speaking. Results. Results from the reviewed research investigations show that they are quite useful for teachers and learners since CA facilitate learners' oral production employing the interaction among classmates and teachers. Conclusion. Besides, Communicative activities encourage learners to develop their language competencies due to the dynamic in which these activities are carried out in the EFL classroom.

\section{Introducción}

La era digital inmersa en las sociedades actuales ha transformado nuestras experiencias y modos de aprender, la globalización y el uso de las nuevas tecnologías de la información y la comunicación, precisa nuevas lógicas cognitivas, la cultura colectiva está cambiando sustancialmente de "estar soportada únicamente por los libros a estar basada también en el ordenador a través de los medios, soportes de información y comunicación que conforman las TIC" (De Velazco \& Chumaceiro, 2016, p. 262); el acceso al internet, la 
virtualidad, la interactividad, el uso de las redes sociales, los video juegos, y la maleabilidad del uso de las Tics, en palabras de Cabero \& Barroso (2016), están alcanzando relevante importancia en los nuevos contextos educativos, su incremento ha facilitado los procesos comunicativos, los procesos de aprendizaje y la cultura, está "cambiando nuestra manera de trabajar, pensar y tomar decisiones" (Garrote et al., 2018, p.2).

Las ideas expuestas sin duda nos llevan a reflexionar sobre la necesidad de una educación de calidad, que sea innovadora y que coadyuve a reforzar los procesos de desarrollo, cultural, social y económico; donde el conocimiento y el aprendizaje sean las claves para enfrentar los desafíos educativos que por la pandemia del COVID-19, han transitado de un aprendizaje pasivo a un aprendizaje activo, los centros de educación en todos los niveles se han visto en la imperiosa necesidad de desarrollar nuevas metodologías, con el fin de garantizar la continuidad en los aprendizajes de niños y jóvenes, debido al cierre de las entidades educativas. Según datos de la Organización de las Naciones Unidas para la Cultura, las Ciencias y la Educación (UNESCO, 2020b) más de 190 países han dado lugar al cierre masivo de cualquier actividad presencial en instituciones educativas con el fin de evitar la propagación del virus y mitigar su impacto. En total, más de 1.500 millones de alumnos afectados, lo que representa al $90 \%$ de la población estudiantil global.

Pero el acceso limitado a internet es uno de los elementos que sin duda traen dificultades para que la educación virtual sea efectiva, los procesos de enseñanza- aprendizaje se ven condicionados al uso de herramientas para el uso y manejo de las tecnologías de la información y la comunicación.

La Organización de las Naciones Unidas para la Cultura, las Ciencias y la Educación (UNESCO, 2020a) ha identificado grandes brechas en los resultados educativos, que se relacionan con una desigual distribución de los docentes, en general, y de los docentes mejor calificados, en particular, en desmedro de países y regiones con menores ingresos y de zonas rurales, las que suelen concentrar además a población indígena y migrante.

La educación virtual, se convierte en una alternativa para la continuidad educativa, sin embargo, esta se ha visto limitada debido a factores económicos, ambientales, políticos y sanitarios, que han retrasado el progreso en la educación de muchos estudiantes.

El impacto que ha causado la presencia del COVID-19 representa como lo dice PachayLópez \& Rodríguez-Gámez (2021):

Una crisis simultánea que ha ocurrido en el mundo entero, evitar la deserción escolar, garantiza una condición escolar saludable; sin embargo, la reducción del aprendizaje es claro y han incrementado los estudiantes desertores, especialmente en grupos vulnerables, acrecentando el trabajo infantil de familias que no lograron vencer la pandemia. (p. 4) 
A pesar del esfuerzo realizado por las instituciones educativas, a raíz de la crisis sanitaria por el COVID-19, la perplejidad en temas de educación se agudiza en todo el mundo; la educación en línea se ha asumido como una educación también de emergencia; es así como:

Los medios digitales se han convertido en una herramienta primordial para la educación virtual, muchas instituciones educativas a nivel mundial han adoptado el proceso de la educación en línea con el único objetivo de mantener sostenible los procesos de aprendizaje, aplicando en la mayoría de los casos un aprendizaje remoto basado en videoconferencias (Valero-Cedeño et al., 2020, p. 4).

Entre las modalidades de aprendizaje a distancia en línea, según La Comisión Económica para América (CEPAL, 2020), enfatiza el uso de plataformas virtuales de aprendizaje asincrónico, utilizadas en 18 países, y también realizan transmisiones de programas educativos por medios de comunicación tradicionales como la radio o la televisión, pero la implementación de estas modalidades en la educación, ahora virtual, se ven involucrados aspectos como la adaptación y priorización del currículo, tomando en consideración los contextos y la pertinencia de los contenidos, igualmente es importante que en estos ajustes prevalezcan las competencias y los valores que se han expuesto como prioritarios en la actual coyuntura: la solidaridad, el aprendizaje autónomo, el cuidado propio y de otros, las competencias socioemocionales, la salud y la resiliencia, entre otros.

El aprendizaje condicionado ahora por la virtualidad tiene que repensar el desarrollo educativo, y plantear didácticas de aprendizaje que comulguen con las nuevas realidades, "el uso de las tecnologías de la información y la comunicación (TIC) no solo provee herramientas, medios, recursos y contenidos, sino, principalmente, entornos y ambientes que promueven interacciones y experiencias de interconexión e innovación educativa" (Varguillas y Bravo, 2020, p.220). También es preciso considerar que la educación virtual requiere de transformaciones que migren las formas de enseñanza tradicional hacia el uso de estrategias educativas virtuales, que además promuevan un dialogo de saberes entre docentes y estudiantes con la consolidación de competencias digitales, por esto es importante según González-Díaz et al. (2020), que docentes y estudiantes se adapten de manera rápida a estos nuevos procesos de enseñanza, que favorezcan encuentros virtuales a través de espacios que traten de mejorar el desarrollo de habilidades comunicativas, tecnológicas y formativas, reconociendo la facilidad remota del estudiante desde cualquier lugar, de manera sincrónica o asincrónica.

Según Ronceros et al. (2021) afirman que "la creación de espacios virtuales en entornos académicos resulta fundamental y necesario, más aún en épocas de confinamiento social, donde cobran fuerza los modelos pedagógicos online desde la virtualidad (e-learning) (p12), la gestión del conocimiento se respalda en las plataformas tecnológicas que existen 
en ambientes académicos propicios para el intercambio de aprendizaje y experiencias en un binomio: Docente-estudiante.

El papel mediador del docente frente a las necesidades de los estudiantes necesita en palabras de Shah et al. (2021), un nexo entre el clima de aprendizaje virtual y el compromiso de los estudiantes, por eso es que establecer una distinción entre la satisfacción de las necesidades y la no satisfacción de las necesidades en términos de sus roles mediadores entre la relación del clima de aprendizaje y el compromiso de los estudiantes es parte de las nuevas prácticas educativas, así como la dimensión de motivación de los estudiantes hacia el aprendizaje en línea durante este tiempo de transición.

\section{Metodología}

La presente investigación tiene un enfoque cuantitativo, tipo de estudio descriptivo porque estudia la percepción de los estudiantes, diseño no experimental, sin manipulación de las variables (Hernández-Sampieri y Torres, 2018), por la recolección de datos en un momento específico es un estudio transversal. La población integra a la totalidad de estudiantes de educación superior de Riobamba - Ecuador (de acuerdo con los datos proporcionados por las instituciones es de 34.000), con un nivel de confianza del $95 \%$ y el error muestra del 5\%, la muestra resultante es de 380. El instrumento se desarrolló de acuerdo con la experiencia de los estudiantes y organizado mediante el modelo diseño instruccional ARCS (Keller, 1987), mediante la técnica de la encuesta online (Google Forms), del 28 de junio al 2 de agosto de 2021, con una escala de tipo Likert, el rango va desde 1 a 5 puntos (1: Total desacuerdo, 2: En desacuerdo, 3: Indiferente, 4: De acuerdo, 5: Total acuerdo), el número de ítems es 36 y dividido en 4 dimensiones (Atención, Confianza, Satisfacción, Relevancia). Para el análisis de los datos se utilizó el SPSS-25 y permitió realizar el análisis de frecuencias.

Fases de investigación:

- Análisis de problemas: Inició con el análisis de los problemas, requerimientos y factores que generan motivación a los estudiantes de la modalidad de educación virtual.

- Revisión de la literatura: En las bases de datos científicas se realizó la revisión de la literatura para comprender el estado de la investigación e identificar los instrumentos de evaluación.

- Rediseño del cuestionario: Con la identificación de los factores que contribuyen a la motivación de los estudiantes y los factores obtenidos en la revisión de la literatura se logrón rediseñar el cuestionario y posteriormente validar con un panel de expertos. 
- Recolección de datos: Para obtener los datos se aplicó la técnica de la encuesta online a los estudiantes.

- Procesamiento y análisis de los datos: Mediante el software SPSS se pudo analizar las frecuencias de acuerdo con las variables analizadas.

- Resultado de la investigación e informe final: Finalmente se obtuvo las conclusiones y se elaboró el informe de investigación.

\section{Resultados}

En muchas investigaciones se analiza la calidad de la educación virtual desde una perspectiva tecnológica, pero en esta investigación se realiza desde un enfoque centrado en los estudiantes, que implica el análisis de la motivación y la activación de la recompensa para cumplir con una acción determinada.

En la tabla 1 se presenta la dimensión 1. Atención, identificada con total acuerdo; en el (ítem 2) las actividades que son gamificadas, juegos o retos para competir con mis compañeros tienen que analizarse con especial cuidado, en el (ítem 12) son importantes también para análisis las actividades prácticas con procesos claros, porque visibilizan el desarrollo de habilidades. En el nivel de acuerdo, respecto al (ítem 1) es de interés el método de aprendizaje basado en proyectos, ya que se puede aplicar los conocimientos, en el (ítem 3) se menciona el aprendizaje colaborativo porque aporta con los conocimientos de los estudiantes y un aprendizaje entre iguales, el (ítem 6) el aprendizaje basado en la resolución de problemas, tiene una gran relevancia para la profesión, (ítem 8) aprender mediante videoconferencias ayuda a mejorar la educación virtual, (ítems 9) los contenidos de los cursos virtuales ayudó a mantener la atención y en el (ítems13), las actividades como videojuegos o entornos inmersivos 3D divierten y ayudan a mantener la atención. En desacuerdo, el (ítem 4) todos los estudiantes son muy responsables y colaboran para realizar los trabajos en grupo, (ítems 5), distracción cuando está prendida la televisión o aparecen las notificaciones de las redes sociales lo que dificulta organizar los tiempos para estudiar, (ítem 10), la comunicación e interacción entre compañeros en la realización de tareas prácticas contribuye para mantener la atención, en el (ítem 14), algunos documentos de texto (PDF) tienen muchas páginas y reducen la atención porque no presentan información relevante. Y en total desacuerdo, el (ítem 11), donde se el buen diseño del aula virtual es también un factor importante.

Tabla 1

Atención

\begin{tabular}{lccccc}
\hline Ítems & 1 & 2 & 3 & 4 & 5 \\
\hline 1. Proyectos & $4,0 \%$ & $12,0 \%$ & $28,0 \%$ & $\mathbf{3 2 , 0 \%}$ & $24,0 \%$ \\
\hline 2. Juegos & $2,0 \%$ & $10,0 \%$ & $26,0 \%$ & $30,0 \%$ & $\mathbf{3 2 , 0 \%}$ \\
\hline 3. Colaborativo & $6,0 \%$ & $6,0 \%$ & $10,0 \%$ & $\mathbf{4 6 , 0 \%}$ & $32,0 \%$ \\
\hline
\end{tabular}


Tabla 1

Atención (continuación)

\begin{tabular}{lccccc}
\hline Ítems & 1 & 2 & 3 & 4 & 5 \\
\hline -4. Responsabilidad & $32,0 \%$ & $\mathbf{4 8 , 0 \%}$ & $14,0 \%$ & $4,0 \%$ & $2,0 \%$ \\
\hline -5. Distracción & $24,0 \%$ & $\mathbf{3 8 , 0 \%}$ & $16,0 \%$ & $18,0 \%$ & $4,0 \%$ \\
\hline 6. Resolver & $4,0 \%$ & $8,0 \%$ & $18,0 \%$ & $\mathbf{5 2 , 0 \%}$ & $18,0 \%$ \\
\hline 8. Videoconferencia & $8,0 \%$ & $12,0 \%$ & $30,0 \%$ & $\mathbf{3 4 , 0 \%}$ & $16,0 \%$ \\
\hline 9. Contenidos & $4,0 \%$ & $8,0 \%$ & $18,0 \%$ & $\mathbf{4 6 , 0 \%}$ & $24,0 \%$ \\
\hline -10. Interacción & $22,0 \%$ & $\mathbf{3 0 , 0 \%}$ & $14,0 \%$ & $20,0 \%$ & $14,0 \%$ \\
\hline -11. Diseño & $\mathbf{3 6 , 0 \%}$ & $22,0 \%$ & $12,0 \%$ & $18,0 \%$ & $12,0 \%$ \\
\hline 12. Habilidades & $6,0 \%$ & $6,0 \%$ & $14,0 \%$ & $22,0 \%$ & $\mathbf{5 2 , 0 \%}$ \\
\hline 13-28. Videojuego & $6,0 \%$ & $4,0 \%$ & $18,0 \%$ & $\mathbf{4 4 , 0 \%}$ & $28,0 \%$ \\
\hline -14. Textos & $22,0 \%$ & $\mathbf{3 2 , 0 \%}$ & $20,0 \%$ & $14,0 \%$ & $12,0 \%$ \\
\hline
\end{tabular}

\section{Fuente: Elaboración propia}

En la tabla 2 se presenta la dimensión 2. Confianza, se observa total acuerdo en el (ítem 17) realizar las actividades prácticas en los cursos virtuales ayudan a desarrollar mis habilidades y reforzar mi confianza en los que estoy aprendiendo, (ítem 20) sentir que mis profesores reconocen mi esfuerzo y me felicitan por realizar buenos trabajos, refuerzan mi confianza en la capacidad que tengo y todo lo que puedo lograr. Los estudiantes están de acuerdo con el (ítem 16), que indica que los contenidos de los videotutoriales tienen buena calidad y refuerzan mi confianza para seguir aprendiendo, el (ítem 19) indica, aprendo con mayor facilidad cuando me explican con ayuda de mapas conceptuales o infografías y refuerza mi confianza en la educación virtual y el (ítem 21) donde se señala que superar los niveles en los videojuegos educativos o entornos inmersivos 3D genera confianza en mi capacidad y me divierte. En desacuerdo con el (ítem 15), que anota disponer de laboratorios virtuales para realizar mis prácticas incrementa mi confianza y el (ítem 18) no se puede trabajar con todos mis compañeros ya que existe opiniones diferentes y reduce mi confianza en los trabajos grupales. En total desacuerdo con el (ítem 22), las clases sincrónicas mediante videoconferencias es difícil comprender y reduce mi confianza en lo que puedo aprender.

Tabla 2

Confianza

\begin{tabular}{lccccc}
\hline Ítems & 1 & 2 & 3 & 4 & 5 \\
\hline -15. Laboratorios & $28,0 \%$ & $\mathbf{3 4 , 0 \%}$ & $12,0 \%$ & $18,0 \%$ & $8,0 \%$ \\
\hline 16. Videotutoriales & $2,0 \%$ & $14,0 \%$ & $26,0 \%$ & $\mathbf{3 8 , 0 \%}$ & $20,0 \%$ \\
\hline 17. Practicas & $4,0 \%$ & $16,0 \%$ & $20,0 \%$ & $24,0 \%$ & $\mathbf{3 6 , 0 \%}$ \\
\hline 18. Grupos & $18,0 \%$ & $\mathbf{3 8 , 0 \%}$ & $14,0 \%$ & $18,0 \%$ & $12,0 \%$ \\
\hline 19. Infografías & $4,0 \%$ & $4,0 \%$ & $12,0 \%$ & $\mathbf{5 8 , 0 \%}$ & $22,0 \%$ \\
\hline 20. Reconocimiento & $6,0 \%$ & $6,0 \%$ & $14,0 \%$ & $22,0 \%$ & $\mathbf{5 2 , 0 \%}$ \\
\hline
\end{tabular}


Tabla 2

Confianza (continuación)

\begin{tabular}{lccccc}
\hline Ítems & 1 & 2 & 3 & 4 & 5 \\
\hline 21. Videojuego & $4,0 \%$ & $8,0 \%$ & $12,0 \%$ & $\mathbf{5 6 , 0 \%}$ & $20,0 \%$ \\
\hline 22. Videoconferencia & $\mathbf{3 6 , 0 \%}$ & $18,0 \%$ & $18,0 \%$ & $16,0 \%$ & $12,0 \%$ \\
\hline
\end{tabular}

Fuente: Elaboración propia

En la tabla 3 se presenta la dimensión 3. Satisfacción, se observa total acuerdo en el (ítem 25), irresponsabilidad: Me desagrada trabajar con algunos compañeros que son irresponsables y el (ítem 27) me divierte el aprendizaje basado en juegos. De acuerdo con el (ítem 23) me gusta las tareas o actividades prácticas que se presenta en los cursos virtuales, el (ítem 24) creatividad: Me llena de satisfacción poder aplicar mi creatividad y sentir que estoy logrando resolver problemas complicados, (ítem 26) luego del esfuerzo empleado para cumplir con las tareas y actividades del aula virtual me llena de satisfacción las calificaciones que obtengo y las felicitaciones que recibo y el (ítem 28) me agrada aprender mediante la realización de proyectos relacionados con mi carrera profesional.

Tabla 3

Satisfacción

\begin{tabular}{lccccc}
\hline Ítems & 1 & 2 & 3 & 4 & 5 \\
\hline 23. Práctica & $4,0 \%$ & $8,0 \%$ & $20,0 \%$ & $\mathbf{4 4 , 0 \%}$ & $24,0 \%$ \\
\hline 24. Creatividad & $6,0 \%$ & $12,0 \%$ & $28,0 \%$ & $\mathbf{3 0 , 0 \%}$ & $24,0 \%$ \\
\hline -25. Irresponsabilidad & $6,0 \%$ & $4,0 \%$ & $12,0 \%$ & $26,0 \%$ & $\mathbf{5 2 , 0 \%}$ \\
\hline 26. Calificaciones & $4,0 \%$ & $6,0 \%$ & $22,0 \%$ & $\mathbf{4 8 , 0 \%}$ & $20,0 \%$ \\
\hline 27. Juegos & $4,0 \%$ & $6,0 \%$ & $18,0 \%$ & $24,0 \%$ & $\mathbf{4 8 , 0 \%}$ \\
\hline 28. Proyectos & $4,0 \%$ & $6,0 \%$ & $16,0 \%$ & $\mathbf{4 8 , 0 \%}$ & $26,0 \%$ \\
\hline
\end{tabular}

Fuente: Elaboración propia

En la tabla 4 se presenta la dimensión 4. Relevancia, se observa total acuerdo en el (ítem 29) son muy relevantes para mí los video tutoriales donde se explica claramente el proceso en corto tiempo y presentan buena calidad audio visual, el (ítem 31) realizar las actividades prácticas con procesos claros es muy relevante para mí ya que siento que estoy desarrollando mis habilidades y el (ítem 36) Es muy relevante para mí cuando mis profesores reconocen mi esfuerzo y me felicitan por realizar buenos trabajos. De acuerdo con el (ítem 32) me gusta el aprendizaje colaborativo, porque puedo aportar con mis conocimientos y también aprendo de mis compañeros cuando realizamos trabajos en grupo, (ítem 34) el contenido multimedia de los cursos virtuales ayudan a comprender la importancia de los conocimientos que se presentan y como se aplicar en la vida real, (ítem 35) la rapidez de acceso a la educación virtual ayuda para que no tenga que gastar tanto 
dinero en viajes y perder tiempo y el (ítem 37) la resolución de problemas tiene una gran relevancia para mi profesión y facilita el aprendizaje mediante una forma práctica. En desacuerdo con el (ítem 30) algunos documentos de texto que se colocan en el aula virtual no son contenidos relevantes para poder aprender. Total desacuerdo con el (ítem 33) los documentos de textos son muy básicos, no ayudan a realizar las actividades prácticas.

Tabla 4

Relevancia

\begin{tabular}{lccccc}
\hline Ítems & 1 & 2 & 3 & 4 & 5 \\
\hline 29. Videotutoriales & $4,0 \%$ & $8,0 \%$ & $16,0 \%$ & $26,0 \%$ & $\mathbf{4 6 , 0 \%}$ \\
\hline -30. Textos & $30,0 \%$ & $\mathbf{4 6 , 0 \%}$ & $12,0 \%$ & $8,0 \%$ & $4,0 \%$ \\
\hline 31. Habilidades & $4,0 \%$ & $8,0 \%$ & $14,0 \%$ & $32,0 \%$ & $\mathbf{4 2 , 0 \%}$ \\
\hline 32. Colaborativo & $6,0 \%$ & $4,0 \%$ & $14,0 \%$ & $\mathbf{5 2 , 0 \%}$ & $24,0 \%$ \\
\hline -33. Textos & $\mathbf{4 2 , 0 \%}$ & $24,0 \%$ & $18,0 \%$ & $10,0 \%$ & $6,0 \%$ \\
\hline 34. Aplicación & $6,0 \%$ & $8,0 \%$ & $18,0 \%$ & $\mathbf{4 2 , 0 \%}$ & $26,0 \%$ \\
\hline 35. Acceso & $4,0 \%$ & $10,0 \%$ & $16,0 \%$ & $\mathbf{5 2 , 0 \%}$ & $18,0 \%$ \\
\hline 36. Reconocimiento & $4,0 \%$ & $2,0 \%$ & $18,0 \%$ & $32,0 \%$ & $\mathbf{4 4 , 0 \%}$ \\
\hline 37. Resolver & $4,0 \%$ & $4,0 \%$ & $14,0 \%$ & $\mathbf{5 4 , 0 \%}$ & $24,0 \%$ \\
\hline
\end{tabular}

Fuente: Elaboración propia

\section{Conclusiones}

- Se identifica a la motivación como el principal factor para estimular el aprendizaje del estudiante en la educación virtual y se combina con la comunicación, la didáctica, la tecnología educativa y contenido multimedia. La motivación integra 4 dimensiones (atención, confianza, satisfacción y relevancia) para activar la percepción de recompensa y que el estudiante cumpla con una acción determinada. Los principales resultados evidencian que hay 4 factores que contribuyen en la motivación de los estudiantes de la modalidad virtual, el primero es la atención, se necesita estimular los sentidos y la captación de la curiosidad prolongada, mediante el cambio de la normalidad (desarrollo de habilidades, gamificadas y juegos). El segundo factor es la satisfacción y se basa en el gusto y recompensas que genera la educación virtual, estimulado principalmente por el aprendizaje lúdico (juegos). El tercer factor es la confianza, se basa en la seguridad de éxito y el control que tienen para mantener el nivel, se incrementa con el desarrollo de actividades prácticas y el reconocimiento del esfuerzo de los estudiantes. El cuarto factor es la relevancia y se basa en la utilidad e interés que 
genera la actividad de aprendizaje, se incrementa con la percepción de logro de las habilidades.

\section{Referencias bibliográficas}

De Velazco, J. J. H. G., \& Chumaceiro, A. C. (2016). Docencia, Ciencia y Tecnología. Un Enfoque desde el Ser y el Hacer Tiempos complejos: educación superior, aprendizaje significativo y el uso del tic. Docencia, Ciencia y Tecnología. Fondo Editorial UNERMB

Cabero, J., \& Barroso, J. (2016). ICT teacher training: a view of the TPACK model/Formación del profesorado en TIC: una visión del modelo TPACK. Cultura y educación, 28(3), 633-663.

La Comisión Económica para América Latina [CEPAL]. (2020). La educación en tiempos de la pandemia de COVID-19.

Garrote Rojas, D., Jiménez-Fernández, S., \& Serna Rodríguez, R. M. (2018). Gestión del tiempo y uso de las TIC en estudiantes universitarios. Píxel-Bit: Revista de Medios y Educación, 53, 109-121.

González-Díaz, R. R., Vásquez, C. E., Hurtado, D. R., y Menacho, A. S. (2020). Plataformas interactivas y estrategias de gestión del conocimiento durante el Covid-19. Revista Venezolana de Gerencia, 25(4), 68-81

Hernández-Sampieri, R., \& Torres, C. P. M. (2018). Metodología de la investigación (Vol. 4). México. D. F DF: McGraw-Hill Interamericana.

Pachay-López, M. J., \& Rodríguez-Gámez, M. (2021). La deserción escolar: Una perspectiva compleja en tiempos de pandemia. Polo del conocimiento, 6(1), 130155.

Keller, J. M. (1987). Desarrollo y uso del modelo ARCS de diseño instruccional. Diario de desarrollo instruccional, 10 (3), 2-10

Ronceros, E. D., Rodríguez, W. J. M., Romero, R. M., \& Sánchez, J. L. A. (2021). Enseñanza virtual en tiempos de pandemia. Revista de ciencias sociales, 27(3), 428-440.

Organización de las Naciones Unidas para la Cultura, las Ciencias y la Educación [UNESCO]. (2020 a). Education: From disruption to recovery. [Online]; (2020a). Acceso 24 de septiembre de 2020. Disponible en: https://bit.ly/3evM4sL. 
Organización de las Naciones Unidas para la Educación, la Ciencia y la Cultura [UNESCO]. (2020b), "Nuevas publicaciones cubanas para enfrentar efectos de la COVID-19 sobre la educación", Oficina de la UNESCO en La Habana [en línea] https://es.unesco.org/news/nuevas-publicaciones-cubanas-enfrentarefectoscovid-19-educacion [fecha de consulta: 12 de junio de 2020]

Shah, S. S., Shah, A. A., Memon, F., Kemal, A. A., \& Soomro, A. (2021). Aprendizaje en línea durante la pandemia de COVID-19: aplicación de la teoría de la autodeterminación en la "nueva normalidad”. Revista de Psicodidáctica.

Valero-Cedeño, N. J., Castillo-Matute, A. L., Rodríguez-Pincay, R., Padilla-Hidalgo, M., Cabrera- Hernández, M. (2020). Retos de la educación virtual en el proceso enseñanza aprendizaje durante la pandemia de Covid-19. Dominio de las Ciencias, 6(4), 1201-1220.

Varguillas Carmona, C. S., \& Bravo Mancero, P. C. (2020). Virtualidad como herramienta de apoyo a la presencialidad: Análisis desde la mirada estudiantil. Revista de ciencias sociales Universidad del Zulia, 26(1), 220-233. 
El artículo que se publica es de exclusiva responsabilidad de los autores y no necesariamente reflejan el pensamiento de la Revista Explorador Digital.

\section{¿Ciencia}

El artículo queda en propiedad de la revista y, por tanto, su publicación parcial y/o total en otro medio tiene que ser autorizado por el director de la Revista Explorador Digital.
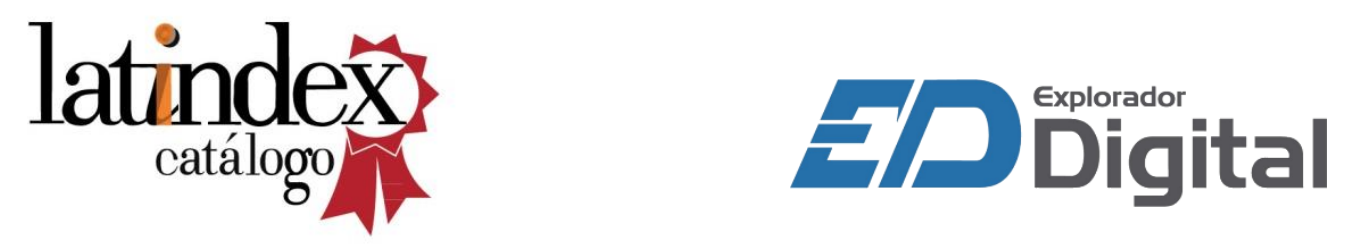

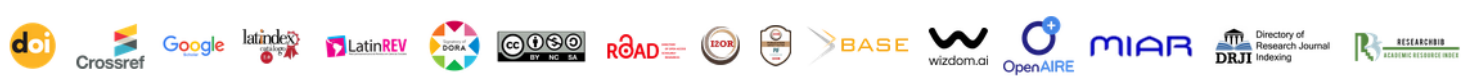
ERIHPLS CCiteFactor 